\title{
ATRIBUIÇÃO DE PRIORIDADES EM REDES DE SENSORES SEM FIO EXPLORANDO MINERAÇÃO DE DADOS EM REDES SOCAIS: IMPLANTAÇÃO E SIMULAÇÃO
}

\author{
Daniel C. Andrade ${ }^{1}$; Daniel G. Costa ${ }^{2}$ e João B. Rocha-Junior ${ }^{3}$ \\ 1. Bolsista FAPESB, Graduando em Engenharia de Computação, Universidade Estadual de Feira de Santana, \\ e-mail: dca650@gmail.com \\ 2. Orientador, Departamento de Tecnologia, Universidade Estadual de Feira de Santana, \\ e-mail: danielgcosta@,uefs.br \\ 3. Colaborador do projeto, Departamento de Ciências Exatas, Universidade Estadual de Feira de Santana, \\ e-mail: joao@uefs.br
}

PALAVRAS-CHAVE: Redes de Sensores sem Fio, Otimização de redes, Mineração de Dados.

\section{INTRODUÇÃO}

As Cidades Inteligentes se tornaram o elemento central para a solução de diversos problemas que atualmente afetam os ambientes urbanos, tais como problemas de mobilidade e eficiência energética que, invariavelmente, são intensificados pelo crescente aumento da população ( $\mathrm{Su}$ et al. (2011)). As iniciativas de Cidades Inteligentes, através da utilização de tecnologias de comunicação e sensoriamento, têm sido desenvolvidas para lidar com a progressiva preocupação com o gerenciamento eficiente de recursos (Costa et al. (2017)). Atualmente, tais iniciativas são baseadas no conceito de Sistema de Sistemas, conjuntura na qual diferentes dispositivos embarcados e inteligentes interagem entre si provendo informações valiosas para a melhoria da qualidade de vida nesses ambientes e, para tanto, operam sobre redes baseadas na Internet ou específicas para a sua aplicação.

A interconexão de sensores na forma de Redes de Sensores Sem Fio (RSSF) permite a criação de infraestruturas distribuídas para o monitoramento das Cidades Inteligentes, possibilitando a coleta de diversas informações que refletem sua dinâmica. A natureza multi-sistema das Cidades Inteligentes traz desafios específicos que requerem soluções inovadoras. Dentre esses desafios, centenas ou milhares de sensores podem estar continuamente transmitindo informação, tarefa que pode requerer uma quantidade considerável de largura de banda e, devido as aplicações envolvidas, se faz necessária uma alta vazão e uma alta taxa de confiabilidade na transmissão. Diante desses requerimentos, a otimização da transmissão dos dados coletados possui um caráter extremamente importante no cenário das Cidades Inteligentes e esta é a motivação principal deste trabalho.

As redes sociais se tornaram bastante populares nos últimos anos devido a proliferação de dispositivos capazes de acessar a Internet (Dafallah (2014)). No Twitter, por exemplo, os usuários compartilham, em tempo real, uma enorme quantidade de dados sobre diferentes tipos de eventos cotidianos (ex: congestionamentos, estréia de filmes). Ao utilizar técnicas de mineração de dados, tais eventos podem ser processados e utilizados para otimizar a transmissão dos dados em uma RSSF (Andrade et al. (2017)). 
Dentro desse contexto, este trabalho dá continuidade à proposta de Andrade et. al (2017) ao propor novos níveis severidade contextual dos eventos, implementar mecanismos de cálculo de escopo e, por fim, efetivar e simular os mecanismos propostos em ambiente computacional.

\section{METODOLOGIA}

O objetivo principal deste trabalho é atribuir níveis de relevância aos nós de uma RSSF a partir da detecção de eventos relevantes no Twitter. O processo completo, definido por Andrade et. al (2017) envolve 3 elementos distintos destacados na Figura 1.

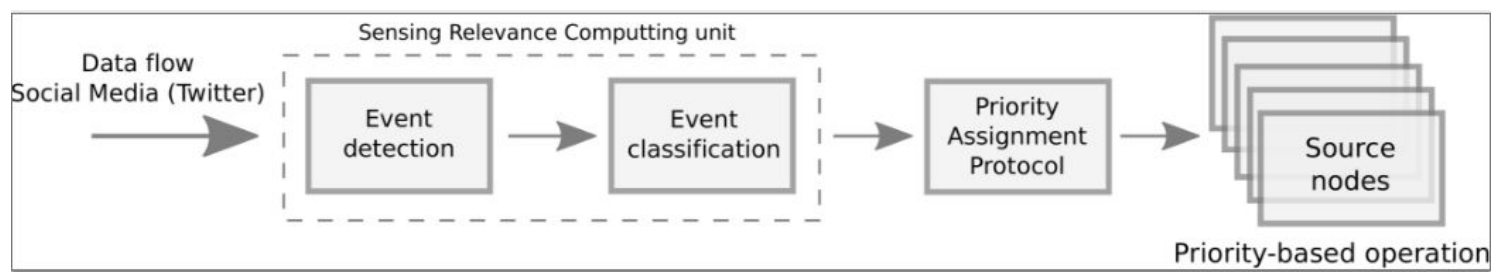

Figura 1: Esquema geral da abordagem

O primeiro dos módulos do sistema, o Detector de Eventos (DE), é responsável por acessar, em tempo real, o fluxo de dados do Twitter e vasculhar por eventos relevantes para a aplicação. Em seguida, os eventos detectados são encaminhados para o Classificador de Eventos (CE) para que tenham seu grau de prioridade extraído e enviado aos nós sensores pelo Protocolo de Definição de Prioridades. Na execução de trabalhos anteriores (Andrade et. al (2017)), o DE foi finalizado e o CE foi arquitetado e implementado considerando apenas a importância do evento segundo a sua natureza, ou seja, a sua severidade contextual. Para propor uma escala mais abrangente, as escalas de severidade foram modificadas e estão descritas na Tabela 1.

Tabela 1. Níveis de severidade contextual.

Severidade contextual Tipo de Evento

Exemplo

$\begin{array}{ccc}100 & \text { Irrestrito } & \text { Grandes protestos } \\ 75 & \text { Social } & \text { Estreia de filme } \\ 50 & \text { Clima } & \text { Chuva } \\ 25 & \text { Mobilidade } & \text { Visita ao zoológico } \\ 0 & \text { Imperceptível } & \text { Eventos rotineiros }\end{array}$

Para incrementar a funcionalidade do CE, o cálculo do escopo deve ser realizado e o valor resultante combinado ao valor da severidade contextual. Para estimar o escopo de um evento, admite-se que uma cidade segue uma rotina semanal e, portanto, a densidade populacional de uma mesma área tende a ser constante nos mesmos dias e horários. Seguindo esta premissa, as mensagens referentes aos dados históricos da cidade são agrupadas em função do dia da semana em qual cada uma foi criada, resultando em sete diferentes grupos de dados. A partir disso, para cada evento, seu dia de publicação é área de abrangência são recuperados e uma consulta espaço-temporal (Andrade et al. (2017)) é executada no grupo correspondente ao seu dia de publicação, visando assim obter uma estimativa média populacional da região do evento. Logo após, dado que diversos eventos podem ocorrer de forma paralela, as médias populacionais da região de cada evento são normalizadas e colocadas numa escala de 0 a 100 , resultando no valor 
do escopo de cada evento. Por fim, a prioridade final do evento é definida como a média aritmética de seu escopo e severidade.

O Protocolo de Definição de Prioridades (PDE), por sua vez, mantém um registro de todos os sensores da rede e suas respectivas prioridades. Ao receber a lista de eventos do CE, acompanhados de sua respectiva área de impacto e prioridade, o PDE calcula quais sensores estão nas áreas do evento e precisam ter sua prioridade atualizada. Para reduzir a quantidade de informações de priorização a serem transmitidas pela rede, apenas os sensores cujas prioridades diferem das novas são atualizados. É importante salientar que as prioridades assimiladas pelos sensores não expiram, sendo somente mudadas quando há instruções diretas emitidas através do PDE.

\section{RESULTADOS}

A solução proposta foi avaliada utilizando um conjunto de dados reais composto por 1.74 mensagens do Twitter obtidas através de sua API pública de streaming. Tais dados representam uma fração de todos as mensagens publicadas no Twitter durante o período de 07/02/2017 e 19/05/2017 na cidade de Nova York (EUA). Os algoritmos construídos foram executados em sua configuração padrão (Andrade et al. (2017)). As Figuras 2 e 3 apresentam os mapas de calor dos eventos detectados ponderados pelas suas respectivas prioridades, plotados através da API do Google Maps ${ }^{1}$. A Figura 2 apresenta os eventos detectados durante as semanas 0 e 1 , demonstrando um alto número de eventos irrestritos ao longo de Manhattan e alguns poucos eventos distribuídos sobre o Brooklyn, tendência que se confirma na Figura 3. Sabe-se que a cidade de Nova York tem uma população desigualmente distribuída, sendo que Brooklyn e Manhattan concentram a maior parte da população e, naturalmente, geram os eventos mais relevantes (Costa et al. (2018)).

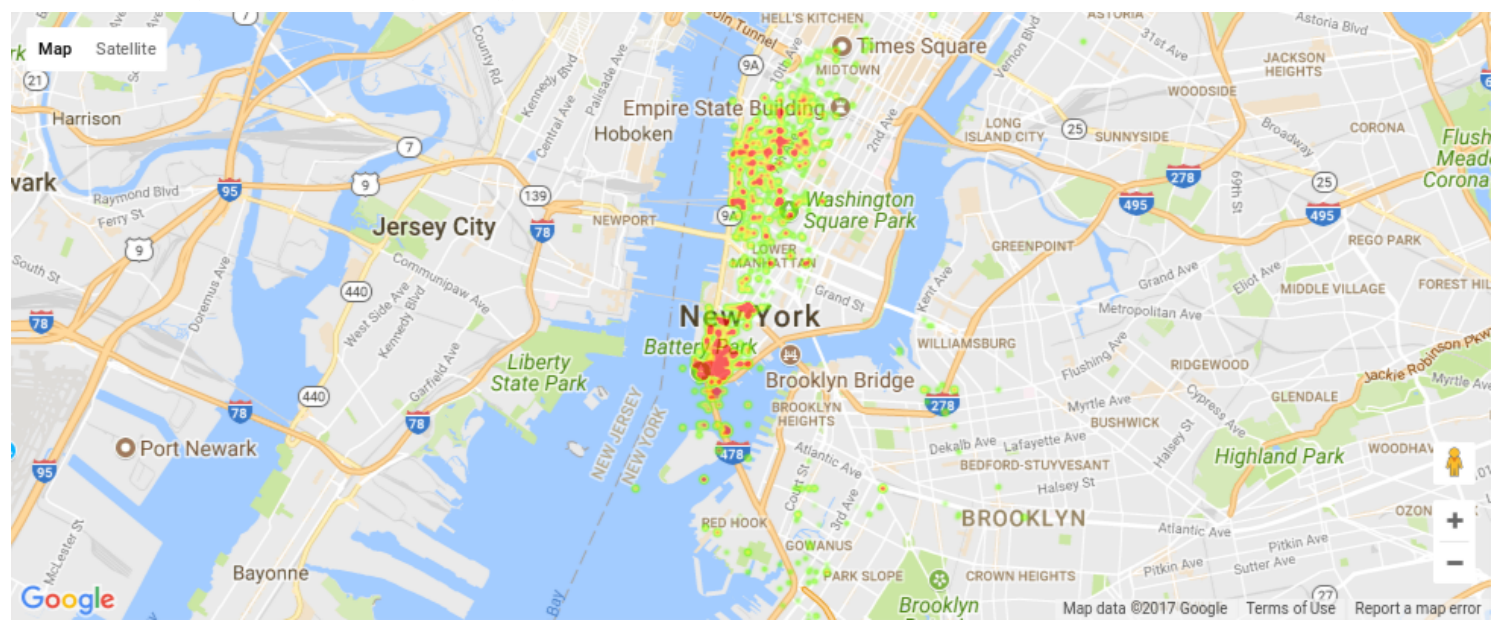

Figura 2: Distribuição das prioridades nas Semanas 0 e 1

De imediato, nota-se uma disparidade considerável entre as prioridades dos eventos nas Figuras 2 e 3, indicando que as Semanas 0 e 1 apresentam eventos muito mais relevantes que as Semanas 6 e 7. Ao analisar os eventos gerados em ambos os casos, percebe-se que o período representado na Figura 2 compreende as manifestações, protestos e shows realizados durante o Mês Histórico das Mulheres, período que os

\footnotetext{
${ }^{1}$ https://cloud.google.com/maps-platform/
} 
Americanos reservam para destacar as contribuições históricas e contemporâneas das mulheres na sociedade. De forma complementar, o período representado na Figura 3 não compreende datas com grandes mobilizações públicas, apresentando assim prioridades mais amenas (Costa et al. (2018)).

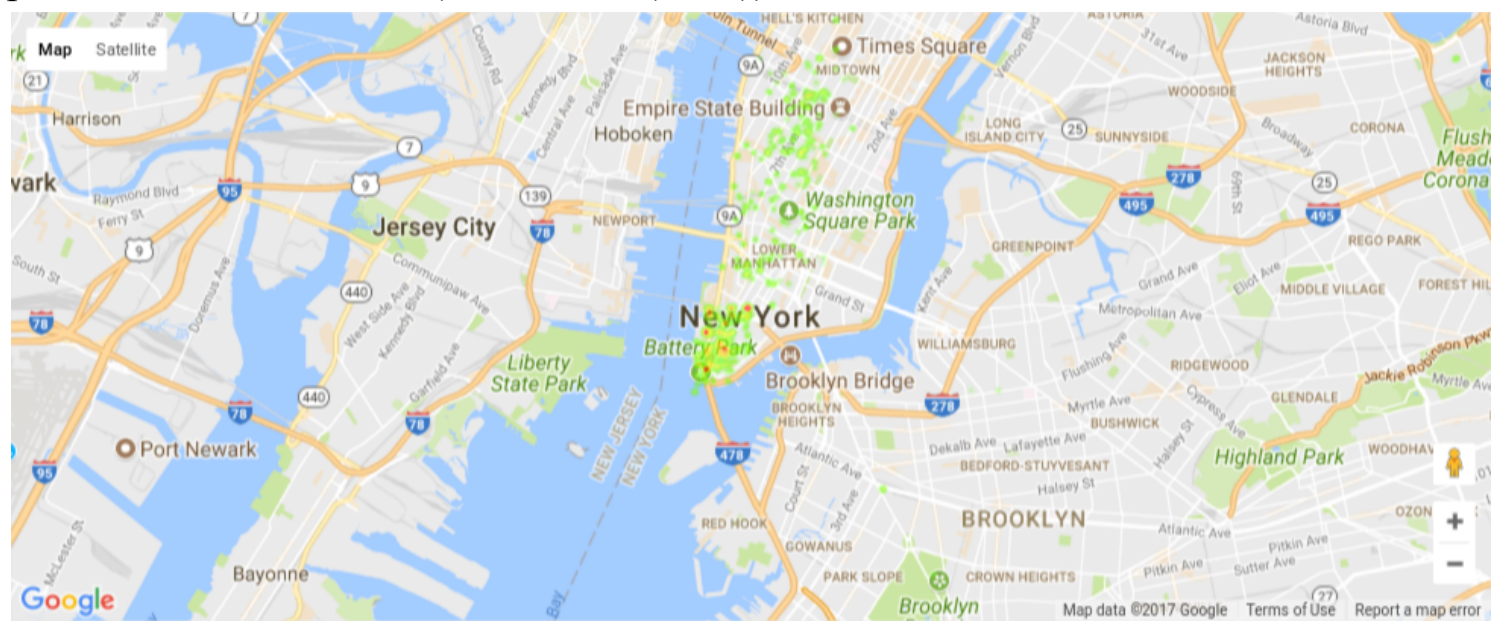

Figura 3: Distribuição das prioridades nas Semanas 6 e 7.

\section{CONSIDERAÇÕES FINAIS}

Em uma RSSF, diversos sensores podem ser implantados para monitorar uma determinada área. Diante desse cenário, é natural supor que informações extraídas da Internet possam ser inseridas em RSSF, permitindo diversas estratégias de otimização na rede. Após a realização dos testes, é perceptível que a exploração de mensagens do Twitter para a priorização de nós de uma RSSF é uma tarefa factível, abrindo novas possibilidades para a otimização do monitoramento das Cidades Inteligentes.

\section{REFERÊNCIAS}

SU, K.; LI, J.; FU, H. Smart city and the applications. In Proceedings of the 2011 International Conference on Electronics, Communications and Control (ICECC), Ningbo, China, 9-11 September 2011;

COSTA, D.G.; COLlOTTA, M.; PAU, G. and DURAN-FAUNDEZ, C., 2017. A fuzzy-based approach for sensing, coding and transmission configuration of visual sensors in smart city applications. Sensors, 17(1), p.93.

DAFALLAH, H.A.A. Design and implementation of an accurate real time GPS tracking system. In Proceedings of the The Third International Conference on e-Technologies and Networks for Development (ICeND2014), Beirut, Lebanon, 29 April-1 May 2014.

ANDRADE, D.C.; COSTA, D.G.; ROCHA-JUNIOR, J.B. Adaptive Sensing Relevance Exploiting Social Media Mining in Smart Cities. In Proceedings of the 23rd Brazillian Symposium on Multimedia and the Web, Gramado, Brazil, 17-20 October 2017.

COSTA, D.G., et al. "TwitterSensing: An Event-Based Approach for Wireless Sensor Networks Optimization Exploiting Social Media in Smart City Applications." Sensors 18.4 (2018): 1080.

ANDRADE, D. C., ROCHA-JUNIOR, J. B., \& COSTA, D. G. Efficient Processing of Spatio-Temporal-Textual Queries. In Proceedings of the 23rd Brazillian Symposium on Multimedia and the Web, Gramado, Brazil, 17-20 October 2017. 